\title{
THE RADIATIVE BIEXCITON-EXCITON RECOMBINATION IN WURTZITE CRYSTALS
}

\author{
W. UNGIER \\ Institute of Physics, Polish Academy of Sciences \\ Al. Lotników 32/46, 02-668 Warszawa, Poland
}

\begin{abstract}
In the effective mass approximation in the two-band model of semiconductor the dipole moment matrix element due to the biexciton-exciton transition is expressed by the functional of the exciton and biexciton envelopes. In comparison to previous estimations of the biexciton-exciton transition probability the detailed band structure at band extrema was taken into account and the biexciton envelope optimized by variational calculation was used in calculations. The oscillator strength ratio $I^{2}=f_{\text {biex-ex }} / f_{\text {band-to-band }}$ is given for several values of $\sigma=m_{e} / m_{\mathrm{h}}$. The calculated functional $I$ for CdS is about four times smaller compared to that obtained by Hanamura. The calculated giant oscillator strength of the biexciton-exciton recombination in CdS is $f_{\text {biex-ex }}=4 \times 10^{2}$.
\end{abstract}

PACS numbers: 71.35.-y

\section{Introduction}

An exciton according to the Wannier-Mott model can be regarded as a bound state of an electron in the conduction band and a positive hole in the valence band. Two weakly bound excitons form a biexciton, similarly to two hydrogen atoms, which can form a hydrogen molecule. In several insulating crystals some of the luminescence lines are interpreted as the lines accompanying the optical conversion of an biexciton to an exciton $[1,2]$. The oscillator strengths of the optical biexciton transitions define the intensity of the luminescence lines, as well as the biexciton lifetime. The oscillator strength of the biexciton-exciton transition gives a considerable contribution to the intensity of lines due to the two-photon direct excitation and recombination of the excitonic molecule $[2,3]$.

Theoretical investigations concerning the biexcitons are usually based on the biexciton's envelope wave function which has to be determined by a variational procedure. The calculation of the oscillator strength of the radiative exciton-biexciton transition expressed with the envelope allows to estimate the radiative contribution to exciton lifetime which is the measured data. 


\section{Electronic states}

In crystals of wurtzite structure, which have an uniaxial symmetry (group $C_{6 v}$ ), the bottom conduction Bloch functions at $k=0$ are of the form

$$
c_{1 / 2}=\phi_{\mathrm{c}}|\uparrow\rangle \quad \text { and } \quad c_{-1 / 2}=\phi_{\mathrm{c}}|\downarrow\rangle
$$

and the top valence Bloch functions at $k=0$ are [4]

$$
v_{3 / 2}=\psi_{1}|\uparrow\rangle \text { and } v_{-3 / 2}=\psi_{-1}|\downarrow\rangle
$$

where $\psi_{ \pm}=(1 / \sqrt{2})\left(p_{x} \pm \mathrm{i} p_{y}\right)$ and $p_{x}, p_{y}$ are the $p$-like functions. The states $|\uparrow\rangle$ and $|\downarrow\rangle$ are the pure spin eigenstates corresponding to $\hbar / 2$ and $-\hbar / 2$ respectively. Because of the anisotropy of the wurtzite crystal, the electron states at $k=0$ are the eigenstates of $J_{z}$ component but not of the total angular momentum. $J$. The biexciton ground state corresponds to the four electron state $\left(J_{z}=0\right)$ at $k=0$ [4] $\frac{1}{2}\left[c_{1 / 2}(1) c_{-1 / 2}(3)-c_{-1 / 2}(1) c_{1 / 2}(3)\right]\left[v_{-3 / 2}(2) v_{3 / 2}(4)-v_{3 / 2}(2) v_{-3 / 2}(4)\right]$,

where 1,3 refer to the two conduction electrons and 2, 4 refer to the two valence electrons. We will consider the only allowed biexciton-exciton optical transition from the biexciton ground state $\left(J_{z}=0\right)$ to the exciton states corresponding to the two-electron states $\left(J_{z}= \pm 1\right)$,

$$
c_{-1 / 2} v_{3 / 2}\left(J_{z}=+1\right) \quad \text { or } \quad c_{1 / 2} v_{-3 / 2}\left(J_{z}=-1\right) \text {. }
$$

We consider the transition to the exciton state $J_{z}=-1$. The case of the transition to the state with $J_{z}=+1$ is analogous. The exciton ground state corresponds to $J_{z}= \pm 2$. The exciton $J_{z}= \pm 1$ energy level is shifted to higher energy by the electron-hole exchange interaction.

Corresponding to Eq. (4), we assume the exciton $J_{z}=-1$ wave function as the linear combination

$$
\left|\mathrm{ex} ; J_{z}=-1, \boldsymbol{K}\right\rangle=\sum_{\boldsymbol{l}-\boldsymbol{k}=\boldsymbol{K}} A_{\boldsymbol{K}}(\boldsymbol{l}, \boldsymbol{k}) a_{c 1 / 2, l}^{+} a_{v 3 / 2, k}|g\rangle,
$$

where $|g\rangle$ is the ground state of $N$ electrons filling the valence band and $\boldsymbol{K}$ is the total wave vector of the free exciton. The operator $a_{c 1 / 2, l}^{+}$creates the electron in the conduction band, in the Bloch state with $J_{z}=1 / 2$ and the wave vector $l$, while $a_{v 3 / 2, k}$ annihilates the electron in the valence band, in the Bloch state with $J_{z}=3 / 2$ and the wave vector $k$ (in the effective mass approximation we replace the periodic parts of the Bloch functions by their values $c_{ \pm 1 / 2}$ and $v_{ \pm 3 / 2}$ at the band extrema).

The Fourier components

$$
A_{K}(l, k)=\frac{1}{V} \iint \mathrm{d}^{3} r_{1} \mathrm{~d}^{3} r_{2} \phi_{K}\left(r_{1}, r_{2}\right) \exp \left(-\mathrm{i} l r_{1}+\mathrm{i} k r_{2}\right),
$$

where the free-exciton wave function is

$$
\phi_{\boldsymbol{K}}\left(\boldsymbol{r}_{1}, \boldsymbol{r}_{2}\right)=\frac{1}{\sqrt{V}} \exp \left(\mathrm{i} \boldsymbol{K} \boldsymbol{R}_{0}^{(\mathrm{ex})}\right) \Phi_{1 s}(r)
$$

( $V$ is the volume of the crystal). Here $\boldsymbol{R}_{0}^{(e x)}=\left(m_{\mathrm{e}} \boldsymbol{r}_{1}+m_{\mathrm{h}} \boldsymbol{r}_{2}\right) / M$ with $M=$ $m_{\mathrm{e}}+m_{\mathrm{h}}$ is the position of exciton centre of mass, $m_{\mathrm{e}}$ and $m_{\mathrm{h}}$ are the electron's and hole's effective masses (we describe the exciton with the optical mass average 
$m_{\mathrm{h}}=3\left(2 m_{\perp}^{-1}+m_{\|}^{-1}\right)^{-1}$, where $m_{\|}$is the hole mass parallel to the $c$ axis and $m_{\perp}$ is the hole mass in perpendicular plane). $\Phi_{1 s}(r)=\exp \left(-r / a_{0}\right) / \sqrt{\pi a_{0}^{3}}$ is the hydrogen-like exciton envelope function of $r=r_{1}-r_{2}$.

The biexciton wave function is assumed in the form of linear combination

$$
\left.\mid \text { biex; } J_{z}=0, K\right\rangle=\sum_{l, k} \sum_{l^{\prime}, k^{\prime}} B_{\mathbf{K}}\left(l, k, l^{\prime}, k^{\prime}\right)\left|J_{z}=0 ; k, k, l^{\prime}, k^{\prime}\right\rangle \text {, }
$$

where the excited states $\left|J_{z}=0 ; l, k, l^{\prime}, k^{\prime}\right\rangle$ correspond to the four-particle state $J_{z}=0(3)$, and which are symmetric with respect to the permutation of the wave numbers $l$ and $l^{\prime}$, as well as to the permutation of $k$ and $k^{\prime}$ :

$$
\begin{aligned}
\mid J_{z}= & \left.0 ; l, k, l^{\prime}, k^{\prime}\right\rangle=\frac{1}{2}\left(a_{c 1 / 2, l}^{+} a_{c-1 / 2, l^{\prime}}^{+}-a_{c-1 / 2, l}^{+} a_{c 1 / 2, l^{\prime}}^{+}\right) \\
& \times\left(a_{v 3 / 2, k} a_{v-3 / 2, k^{\prime}}-a_{v-3 / 2, k} a_{v 3 / 2, k^{\prime}}\right)|g\rangle,
\end{aligned}
$$

The summation over $l, k, l^{\prime}$ and $k^{\prime}$ in Eq. (8) is limited to $l-k+l^{\prime}-k^{\prime}=K$, the total momentum of the biexciton.

The linear coefficients $B_{\boldsymbol{K}}$ in Eq. (8) are the Fourier components

$$
\begin{aligned}
& B_{\boldsymbol{K}}\left(\boldsymbol{l}, k, l^{\prime}, k^{\prime}\right)=\frac{1}{V^{2}} \iiint \int \mathrm{d}^{3} r_{1} \mathrm{~d}^{3} r_{2} \mathrm{~d}^{3} r_{3} \mathrm{~d}^{3} r_{4} \psi_{\boldsymbol{K}}\left(\boldsymbol{r}_{1}, \boldsymbol{r}_{2}, r_{3}, r_{4}\right) \\
& \quad \times \exp \left(-\mathrm{i} l \boldsymbol{r}_{1}+\mathrm{i} k r_{2}-\mathrm{i} l^{\prime} r_{3}+\mathrm{i} k^{\prime} r_{4}\right),
\end{aligned}
$$

where $\psi_{K}$ is the biexciton wave function of electron $(1,3)$ and hole $(2,4)$ coordinates

$$
\psi_{\boldsymbol{K}}\left(\boldsymbol{r}_{1}, r_{2}, r_{3}, \boldsymbol{r}_{4}\right)=\frac{1}{2 \sqrt{V}} \exp \left[\mathrm{i} \boldsymbol{K} \boldsymbol{R}_{0}^{(\text {biex })}\right] \Psi\left(r, r^{\prime}, \boldsymbol{R}\right) .
$$

Here $\boldsymbol{R}_{0}^{(\text {biex })}=m_{\mathrm{e}}\left(\boldsymbol{r}_{1}+r_{3}\right) / 2 M+m_{\mathrm{h}}\left(\boldsymbol{r}_{2}+r_{4}\right) / 2 M$ is the position of biexciton's centre of mass and $\Psi$ is the biexciton's envelope function of $r=r_{3}-r_{1}, r^{\prime}=r_{4}-r_{2}$ and $\boldsymbol{R}=\left(r_{1}+r_{3}-r_{2}-r_{4}\right) / 2$. We assume that $\Psi$ corresponds to the ground state energy of biexciton. The factor $1 /(2 \sqrt{V})$ in Eq. (11) was introduced to satisfy the normalization condition $\int \mathrm{d}^{3} r \int \mathrm{d}^{3} r^{\prime} \int \mathrm{d}^{3} R|\Psi(r, r, \boldsymbol{R})|^{2}=1$.

\section{Oscillator strength}

For the transition with emission of light we use, for wurtzite crystals, the customary dipole approximation

$$
\sum_{j=1}^{N} p_{i}=\sum_{\lambda}\left(\left\langle\psi_{1}|p| \phi_{c}\right\rangle a_{v 3 / 2, \lambda}^{+} A_{c 1 / 2, \lambda}+\left\langle\psi_{-1}|p| \phi_{c}\right\rangle a_{v-3 / 2, \lambda}^{+} a_{c-1 / 2, \lambda}\right),
$$

where $\boldsymbol{p}_{j}$ is the momentum of $j$-th electron and $\left\langle\psi_{ \pm 1}|p| \phi_{\mathrm{c}}\right\rangle$ is the matrix element of the band-to-band transition. For the biexciton and exciton states given by Eqs. (5) and (8), for small value of the wave vectors $\boldsymbol{K}, \boldsymbol{K}^{\prime}$ close to zero, we find

$$
\left\langle\text { ex; } J_{z}=-1, \boldsymbol{K}^{\prime}\left|\sum_{j=1}^{N} p_{j}\right| \text { biex; } J_{z}=0, \boldsymbol{K}\right\rangle=-\delta_{\boldsymbol{K}^{\prime} K}\left\langle\psi_{1}|p| \phi_{c}\right\rangle I,
$$

where $I$ is the functional of the exciton's and biexciton's envelopes

$$
I=\iint \mathrm{d}^{3} x \mathrm{~d}^{3} y \Phi_{1 s}^{*}(y) \Psi(x, x-y, y / 2) .
$$


The oscillator strength of the biexciton-exciton transition can be expressed by [5]

$$
f_{\text {biex-ex }}=f_{\text {band-to-band }}\left(\omega_{\mathrm{g}} / \omega\right) I^{2},
$$

where $f_{\text {band-to-band }}$ is the oscillator strength of the valence-band-to conduction-band transition at the center of the Brillouin zone, $\hbar \omega_{\mathrm{g}}$ is the band-gap energy and $\hbar \omega=E^{(\text {biex })}-E^{(\text {ex })}$ is the energy difference between the biexciton's and exciton's states. For shallow excitons we can use the approximation $\omega \approx \omega_{\mathrm{g}}$.

$f_{\text {band-to-band }}$ may be evaluated from the measured value of $f_{\text {ex }}[5]$ (the free-exciton oscillator strength per molecule)

$$
f_{\text {band-to-band }}=f_{\text {ex }}\left(\omega_{\mathrm{ex}} / \omega_{\mathrm{g}}\right) \Omega^{-1}\left|\dot{\Phi}_{1 s}(0)\right|^{-2},
$$

where $\Omega$ is the volume of a unit cell of the crystal.

\section{Results and discussion}

In our calculations the biexciton envelope $\Psi=\psi F$ was approximated by the Hylleraas-Ore wave function $\psi[6,7]$ multiplied by $F$ - the function introduced by Brinkman et al. [8]. The variational parameters of $\Psi$ depend on the ratio of effective masses $\sigma=m_{\mathrm{e}} / m_{\mathrm{h}}$. We have calculated the functional $I$ for few values of $\sigma: I(\sigma=0.1)=7.3, I(\sigma=0.2)=10$, and $I(\sigma=0.3)=12.7$.

In the case of CdS our result for $I$ is about four times smaller compared to that obtained by Hanamura $\Phi_{1 s}(0) g(0)=8 \sqrt{a_{\mathrm{B}}{ }^{3} / a_{0}^{3}} \approx 40$, where $g(k)$ is the Fourier component of the wave function describing the relative motion of the two excitons with the average distance $a_{\mathrm{B}}$ [3]. The result obtained by Hanamura is applied in subsequent publications [2], but attention should be paid to the some inaccuracy of its derivation: (1) The realistic form of the electron wave functions at band extrema was not taken into account. (2) The biexciton state was described by the product of fermion operators without spin indices. It causes that the envelope function containing antisymmetric spatial part was taken into account and the envelope containing symmetric spatial part, determining the binding of excitonic molecule, was neglected. (3) The form of biexciton envelope was chosen, in some sense, arbitrarily, i.e., it was not optimized by variational calculation.

Using for CdS $\sigma=0.21, \Omega=49.4 \AA^{3}$, the exciton Bohr radius $a_{0}=29 \AA$ and $f_{\text {ex }}=2.56 \times 10^{-3}$ [9], we obtained for the oscillator strength of the radiative biexciton-exciton recombination the value $f_{\text {biex-ex }}=4 \times 10^{2}$, which corresponds to the biexciton radiative lifetime $\tau_{\mathrm{biex}-\mathrm{ex}}=8.7 \mathrm{ps}$. Our result is in good agreement with the observed time of biexcitons decay $\tau$, which amounts to few picoseconds [10]. Since other channels of the biexciton decay do exist, one should always have $\tau_{\text {biex-ex }}>\tau$. Previous estimations of the oscillator strengths corresponded to $\tau_{\text {biex-ex }}=0.5 \mathrm{ps}$, which is certainly too small.

\section{Acknowledgments}

The author gratefully acknowledges Prof. M. Suffczyński and Dr. P. Janiszewski for helpful discussions. 


\section{References}

[1] S. Shinoya, H. Saito, E. Hanamura, O. Akimoto, Solid State Commun. 12, 223 (1973).

[2] I.S. Gorban, I.M. Dmitruk, O.A. Yeshchenko, Solid State Commun. 98, 941 (1996).

[3] E. Hanamura, Solid State Commun. 12, 951 (1973).

[4] F. Bassani, J.J. Forney, A. Quattropani, Phys. Status Solidi B 65, 591 (1974).

[5] C.H. Henry, K. Nassan, Phys. Rev. B 1, 1628 (1970).

[6] E.A. Hylleraas, A. Ore, Phys. Rev. 71, 493 (1947).

[7] W. Ungier, M. Suffczyński, J. Quantum Chem. 60, 1001 (1996).

[8] W.F. Brinkman, T.M. Rice, B. Bell, Phys. Rev. B 8, 1570 (1973).

[9] D.G. Thomas, J.J. Hopfield, Phys. Rev. 116, 573 (1959).

[10] A. Hoffmann, private communication. 